\title{
Influences of Planets on Parent Stars: Abundances
}

\author{
Norman Murray \\ Canadian Institute for Theoretical Astrophysics, University of Toronto, \\ 60 St. George st., Toronto, ON, M5S 3H8, Canada; Canada Research \\ Chair in Astrophysics
}

\begin{abstract}
The heavily cratered face of Mercury and the Moon bears mute testimony to the flux of smaller objects that bombarded the inner solar system in the first billion years after the sun formed. A significant fraction of these bodies likely struck the sun, slightly altering its surface abundances of heavy elements. Stellar pollution of this sort can be detected by looking at metallicities of carefully selected populations of stars of varying mass, e.g., in moderate age clusters, by searching for specific patterns in relative abundances in individual stars, or by looking for isotopic anomalies such as ${ }^{6} \mathrm{Li}$. I describe the current state of such studies.
\end{abstract}

\section{Introduction}

By pointing out that the Earth was not at the center of the solar system, Copernicus began the long process of placing man in his proper context in the universe. We now know that we orbit a fairly common type of star on one of several terrestrial bodies. We know that solar-type stars are fairly common, but how common are terrestrial bodies?

We know of one other system in which terrestrial mass bodies orbit a star, the pulsar planet system (Wolszczan \& Frail 1992). However, the rather inhospitable nature of a pulsar makes the system very different from our own. We also know of roughly 100 solar type stars orbited by Jupiter mass planets. All but one of these systems were discovered by radial velocity searches, which are currently not sensitive enough to detect Earth mass planets. Two Jupiter mass extrasolar planets have been seen to transit their parent stars. Transits are a promising method to discover Earth mass planets, but that promise is still years from being fulfilled.

The Copernican assumption, when combined with some knowledge of bodies in our own system, suggests an indirect method for estimating the fraction of solar type stars possessing terrestrial companions. There is evidence that up to an Earth mass of terrestrial material has accreted onto the sun. I will quickly summarize this evidence, then discuss ways of detecting similar accretion in other systems, and describe some of the consequences for the parent stars.

I remind the reader that a solar mass star has a radiative interior surrounded by a surface convection zone. Material dropped onto the surface of the star will be mixed throughout the convection zone within months to years. On longer 
time scales, it will be mixed somewhat deeper into the star, as evidenced by the depletion of lithium seen in the sun and other stars. Figure 1 shows the mass of the surface convection zone as a function of stellar age for stars of different masses. One point to bring away from this figure is that accretion has to take place 30Myrs or so after the star forms if the metallicity of the surface layers is to be altered substantially. A second point is that more massive stars have less massive convection zones, so that the effects of accretion will show up most dramatically in stars with masses comparable or larger than that of the sun.

\section{Solar Accretion}

The Earth and other terrestrial planets and moons are believed to have formed from smaller bodies known as planetesimals. These planetesimals formed in a gas disk surrounding the sun, a disk similar to those seen around young stars in Orion and other star forming regions. The orbits were likely to be dynamically cold, at least initially, so most of the material in the inner solar system likely ended up in one of the current day planets. However, bodies outside the orbit of Mars might have evolved differently, due to the effects of Jupiter's gravitational perturbations. This region is currently occupied by the asteroid belt, which is believed to be the remnant of a once much more massive population of planetesimals.

The current mass of the asteroid belt is of order $5 \times 10^{-4} M_{\oplus}\left(M_{\oplus}\right.$ is the mass of the Earth, about $6 \times 10^{27}$ grams). Estimates of the primordial mass of the belt, based on a smooth extrapolation of the current surface density of metals, are of the order of one to a few Earth masses. Rough estimates of the amount of mass required to allow formation of the asteroids via accretion of smaller $(\sim 1 \mathrm{~km})$ bodies in the available time before the formation of Jupiter also suggest a much larger initial mass for the belt.

Thus it appears that one to a few Earth masses of terrestrial material has been removed from the region between Mars and Jupiter. The removal mechanism was almost certainly gravitational, either by interactions with Mars size objects that were themselves later ejected (e.g., Petit Morbidelli \& Chambers 2001) or by secular or resonant interactions with the giant planets. Objects with initial semimajor axes larger than half that of Jupiter likely were ejected from the solar system by Jupiter. Objects with smaller semimajor axes, on the other hand, most likely were removed by striking the sun.

If this solar accretion did occur, it would have increased the metallicity $([\mathrm{Fe} / \mathrm{H}])$ of the solar convection zone by 0.01 to 0.03 dex $(3$ to $7 \%)$ over that of the radiative interior. One could in principle detect this metallicity difference by measuring the run of sound speed using helioseismology. The slightly lower opacity in the radiative zone would produce variations in sound speed profile in the radiative interior of the sun compared to a uniform metallicity solar model. Currently the best helioseismology based upper limits on the amount of accreted material are of order $10 M_{\oplus}$ (Henney \& Ulrich 1998; Winnick et al. 2002). Alternately a careful measurement of the solar neutrino flux would allow a determination of the central temperature of the sun, but the precision required is not currently available. 


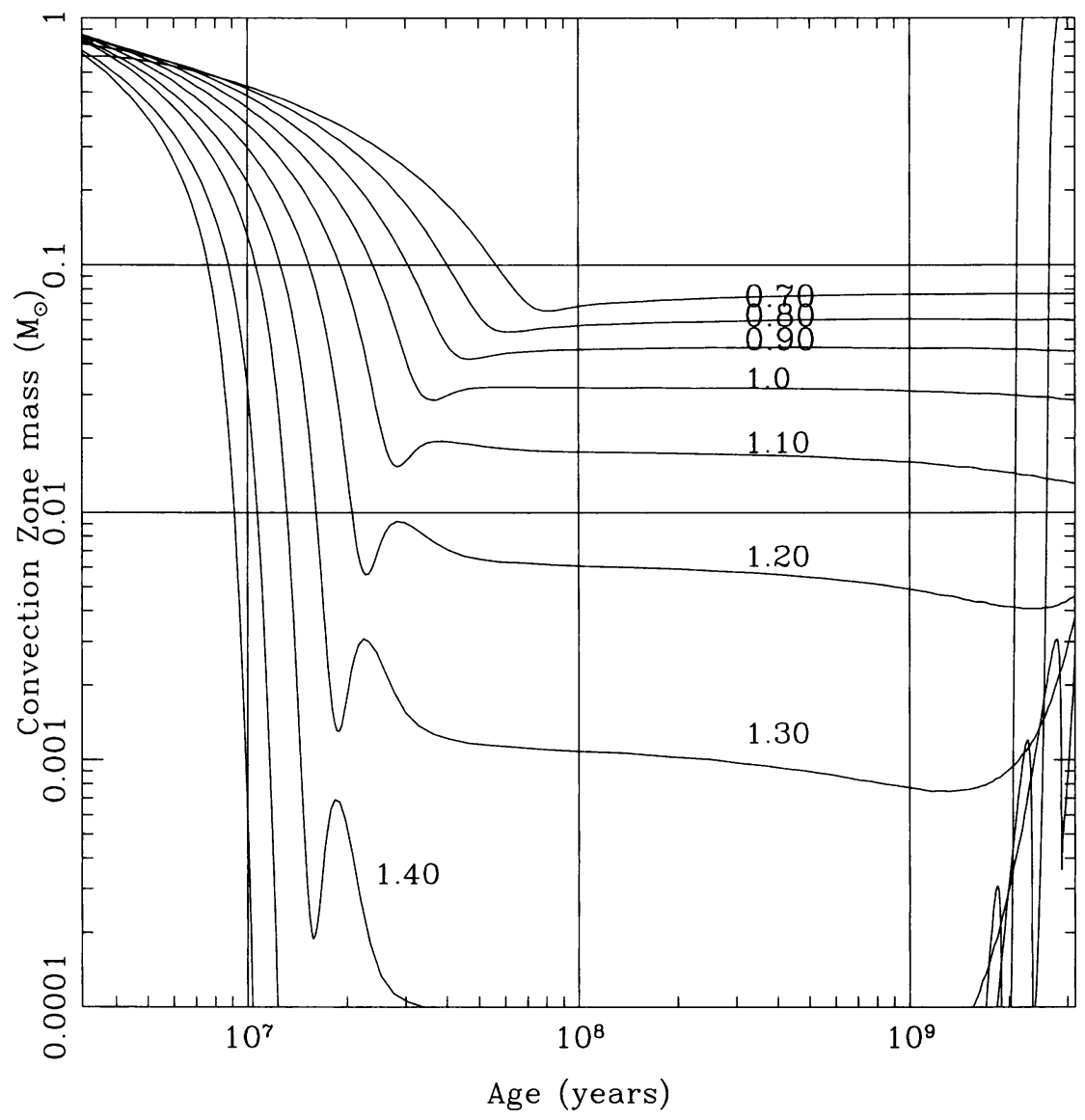

Figure 1. The mass of the convection zone for solar metallicity $([\mathrm{Fe} / \mathrm{H}]=0)$ stars, plotted as a function of stellar age. The stellar mass is used to label the curves. Note that for ages less than about 7 million years all stars in the mass range $0.6-1.6 M_{\odot}$ have convection zone masses greater than $0.1 M_{\odot}$; stars this young have not yet reached the main sequence. 




Figure 2. The average infrared excess $\left(L_{i r} / L_{*}\right)$ of stars in young clusters, as a function of cluster age. Figure from Spangler et al. (2001).

\section{Accretion Onto Other Stars}

There is some evidence that heavy elements are raining down on main sequence stars in the solar neighborhood. The evidence is the sporadic appearance of narrow transient absorption lines in the spectra of stars like Beta Pictoris. One possible interpretation is that these events represent the coma of evaporating comets seen against the disk of the star (Ferlet, Vidal-Madjar \& Hobbs 1987). Beta Pic is known to possess a dust disk which is warped. One possible warping mechanism is the gravitational influence of a Jupiter mass body orbiting the star. Such a planet, if it exists, could also perturb planetesimals onto star grazing orbits. Even if on does not accept this interpretation, there is strong evidence of metal ions falling toward the star at high velocity. This material may be accreting from a circumstellar disk, but it will still enhance the metallicity of the star, as it is clearly hydrogen poor.

Figure 2, taken from Spangler et al. (2001), shows the infrared excess in nearby star clusters as a function of cluster age. The dust in these disks is large (compared to the dust found in the interstellar medium) and is believed to be 
the result of collisional grinding of planetesimals. The dust mass (or IR excess) may decrease because the planetesimals are accreted onto planets, or ejected from the system, or because they are accreted onto the central star. While it is difficult to ascertain the relative fractions, it is at least plausible that a substantial amount of material may hit the star.

\section{Effects of Accretion on the Star}

Now I turn to the effects of this (possible) accretion on the properties of the accreting star. First, if the accretion takes place after the star has reached the main sequence, it will enhance the metallicity of the surface layers over that of the bulk of the star. The amount of the change depends both on the amount of accreted material and on the mass of the convection zone.

Secondly, the accreted material will alter the opacity of the surface layers, and thereby the stellar radius, luminosity, and effective temperature. The effects are very small. The stellar radius increases by about a part in a thousand for $10 M_{\oplus}$ of accreted solids, while the luminosity decreases by a similar amount. The mass of the convection zone increases when solids are added to a solar mass star, but it decreases when solids are added to a 1.2 solar mass star.

Detecting such accretion-induced changes is not trivial. One cannot tell by measuring the surface iron abundance that a star has accreted material. The changes in luminosity and effective temperature produced by accretion can be mimicked by variations in stellar mass or age. How then can we tell if accretion has occurred?

One way is to look for systematic changes in abundances of volatile versus refractory elements. Since the accreted material tends to be highly enhanced in refractory elements such as iron and nickel, while volatiles such as nitrogen and carbon are not so highly enhanced, one can look for trends of increasing abundance as a function of condensation temperature. A few such studies have been carried out for stars with planet, with conflicting results (Smith, Cunha \& Lazzaro 2001; Takeda et al. 2001).

Another test is to look at stars believed to have been formed at the same time out of the same material. If the amount of accretion varies from star to star, then abundances will also vary from star to star. Gratton et al. (2001) and Laws \& Gonzalez (2001) have carried out very careful differential abundance determinations for a handful of binary systems, finding small but apparently significant (three sigma) differences between HD $219542 \mathrm{~A}$ and B, and between 16 Cygni $A$ and $B$. The differences are at the 0.03 dex level. This seems to me to be the best evidence currently available showing that accretion has occurred in some stars.

A variation of this test is to examine stars in clusters. This has been done by Paulson et al. (2003) for the Hyades. They find very little scatter in $[\mathrm{Fe} / \mathrm{H}]$, above their instrumental limit of 0.04 dex. They do find two stars, vB 1 and vB 2, that have significantly higher $[\mathrm{Fe} / \mathrm{H}]$, by 0.2 dex (see Figure 3 ). These stars are not currently bound to the Hyades cluster. However, the cluster is clearly evaporating, as expected theoretically, and the two stars may well have been bound to the cluster when they formed. If the high metallicity is a result of accretion, these two stars would not have accreted much material; at 


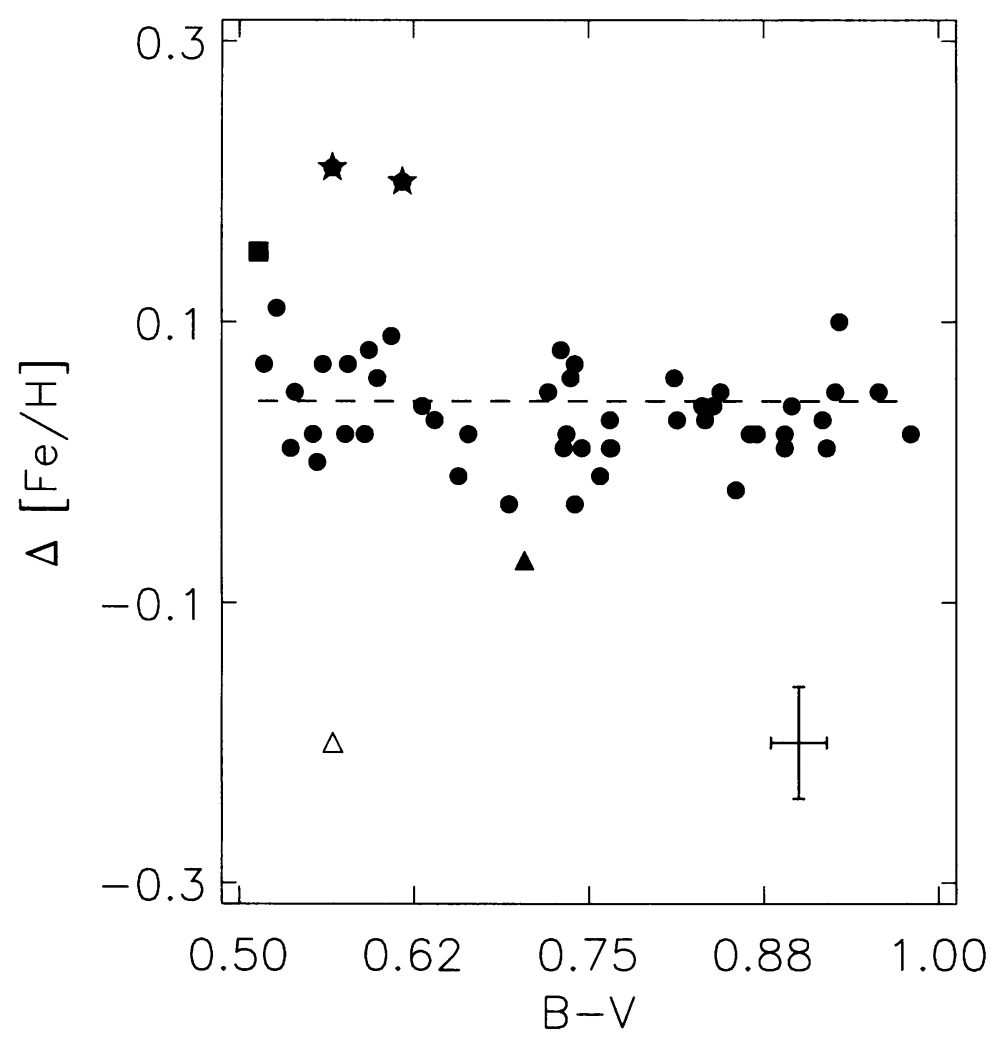

Figure 3. The differential metallicity $[\mathrm{Fe} / \mathrm{H}]-\langle[\mathrm{Fe} / \mathrm{H}]\rangle$ for Hyades stars as a function of stellar color, B-V. The two filled stars represent vB 1 and vB 2. Figure from Paulson et al. (2003). 
$[\mathrm{Fe} / \mathrm{H}]=0.3$ and with effective temperatures in excess of $6000 \mathrm{~K}$, they have very thin convection zones. It would be interesting to measure the radial velocities of these stars, although they are almost certainly very active and fairly rapidly rotating.

A third test is to look for the presence of lithium 6 , the very fragile isotope of lithium. It is completely destroyed in premain sequence solar mass stars of solar metallicity, so the presence of lithium 6 in a main sequence star would provide a smoking gun for accretion. Israelian et al. (2001; 2003) reported the detection of lithium 6 in HD 82943, a star with (at least) two radial velocity planets. Reddy et al. (2002) examined HD 82943 along with several other stars, finding only very low upper limits on the abundance of lithium 6 for all their stars. More recently, Mandell, Ge \& Murray (2003) have examined lithium 7 poor stars, including HD 209458, one of the stars observed by Reddy et al., and found no evidence for the presence of lithium.

While the lithium 6 test offers the promise of a clear detection of accretion, it is clear that it is a difficult measurement. It must also be pointed out that lithium 7 is depleted on the main sequence, so it is likely that lithium 6 is depleted soon after it is accreted. Thus a non-detection is not strong evidence against accretion.

Each of the methods described above examines individual stars, possibly in comparison to others, to look for evidence of accretion. One can also look for statistical evidence of accretion. The idea is to use variations in the mass of the surface mixing layer of stars to search for systematic variations in metallicity. In a first approximation, more massive stars have less massive accretion zones. If accretion is common, one would expect that more massive stars would have higher metallicity than less massive stars, at least on average.

On closer examination this effect is modified. We know from studies of lithium abundances by Boesgaard \& Tripicco (1986) and others that there is some unknown process, probably related to differential rotation, that mixes material from the surface of 1.1-1.3 solar mass stars down into the stellar interior. Stars in this mass range effectively have deeper "convection zones", or rather surface mixing layers, than stars that are either more or less massive. Thus the trend of metallicity is modified; one expects to find that stars in this mass range have lower metallicities than slightly more or less massive stars, assuming accretion has occurred.

It is actually fortunate that this second mixing process exists, for it allows us to rule out a different explanation of increasing metalicity with increasing stellar mass, namely that more massive stars are younger on average, and hence more metal rich, than older, less massive stars, since the metallicity of the galaxy is increasing with time.

Figure 4 shows the average metallicity of a subset of stars in the Cayrel de Strobel catalog as a function of stellar mass, binned by mass (Murray et al. 2001). There is some hint of the expected pattern (shown by the solid line) suggesting that some accretion has occurred in some or all of these stars.

The difficulty with this sample is that it is very heterogeneous; it is a compilation of stellar samples taken from the literature. The selection criteria for the original samples is difficult to ascertain. Some selection criteria can mimic the signature of accretion. For example, many samples are chosen to have B-V 


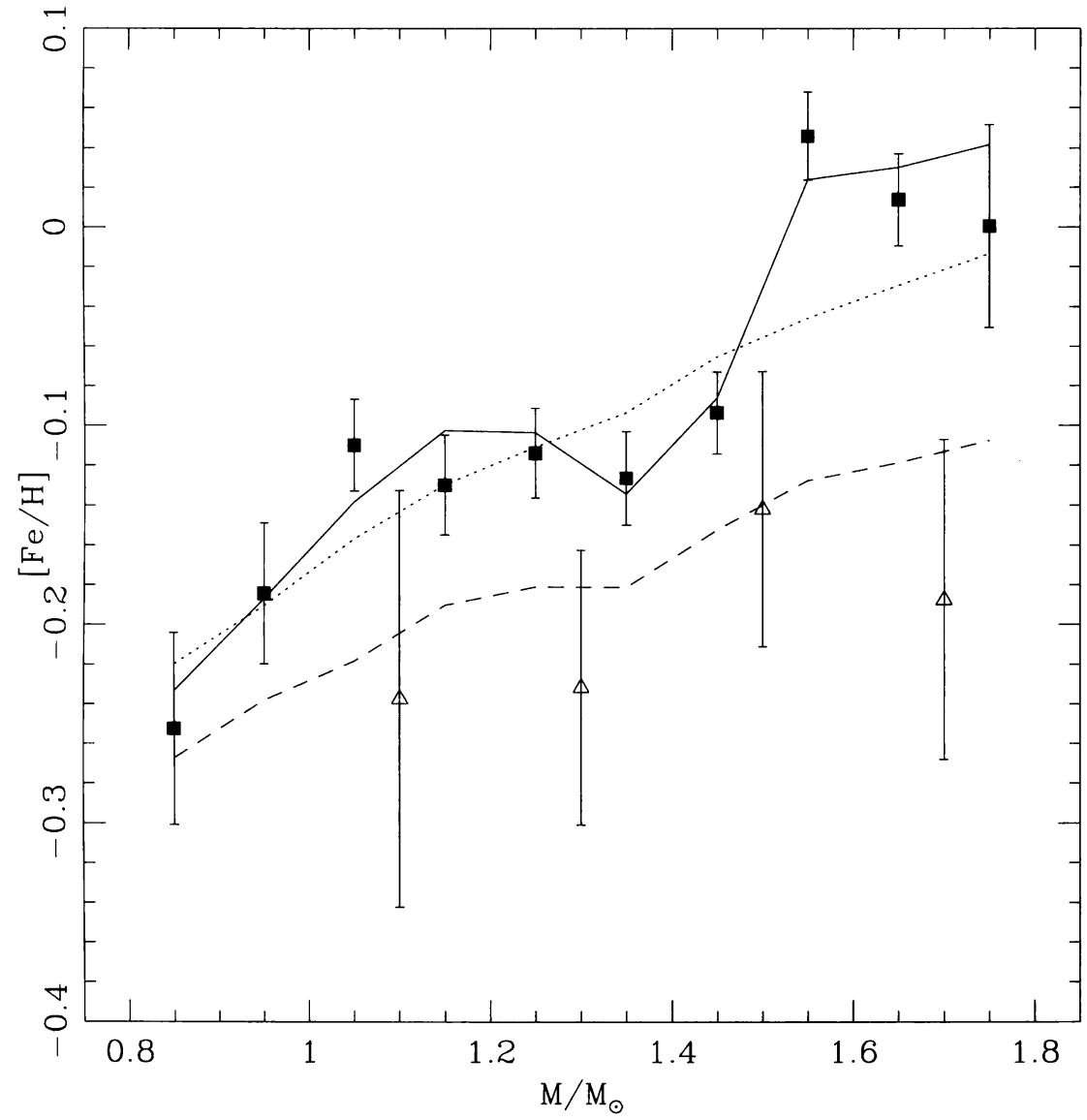

Figure 4. Average stellar metallicity in mass bins of width $0.1 M_{\odot}$ (filled squares, representing unevolved stars). There are roughly 45 such stars per bin. The open triangles represent the evolved stars (dwarf stars with surface convection zones ten or more times larger than $M_{m i x}$. There are only 3-6 stars per bin, with a bin width of $0.20 M_{\odot}$ for these objects. The evolved stars appear to have lower metallicity than unevolved stars of the same mass. The dotted line is an unpolluted Monte Carlo model giving the smallest reduced $\chi^{2}=3.2$. The solid line is a polluted Monte Carlo model with an accreted iron mass of $0.4 M_{\oplus}$ and an age-metallicity slope of -0.14 ; the fit has a reduced $\chi^{2}=1.0$ The dashed line is the polluted model after the mass of the surface mixing region has increased by a factor of ten from its main sequence value, and should be compared to the evolved star data represented by the open triangles. This fit also has a reduced $\chi^{2}$ of order unity. 
colors either in some range, or redder (larger) than some value. Since more massive stars are bluer for a fixed metallicity than less massive stars, requiring $B-V>0.5$, for example, will introduce a trend of increasing metallicity with increasing mass.

It is not clear what kind of selection criteria would produce the pattern seen in Figure 4 (solid squares), but the possibility is there. A sample of hundreds of solar mass stars with known selection criteria would be very informative.

A second statistical technique, also illustrated in Figure 4, relies on the fact that as stars begin to evolve off the main sequence their convection zones deepen. Their surface metallicities then reflect their bulk metallicity. If accretion is common, then these slightly evolved stars should be deficient in metals compared to slightly younger stars of the same mass (on average). The effect is seen in the plot.

\section{Conclusions}

There is moderately compelling observational evidence that accretion is occurring in young main sequence stars such as Beta Pictoris. There is also good evidence that similar accretion occurred in our solar system, at the level of one Earth mass of solid material.

The evidence for widespread accretion of a one to a few Earth masses of refractory material onto main sequence solar type stars is less compelling. The best such evidence comes from studies of binary stars. The results for the Hyades of Paulson et al. suggest that such accretion has not occurred in that cluster, with the possible exception of $\mathrm{vB} 1$ and $\mathrm{vB} 2$, the two stars whose cluster membership is uncertain. The results of Murray et al. are tantalizing, but fraught with unknown selection effects.

The Hyades are currently much less rich than the Orion cluster; however, when the Hyades were forming they were most likely comparable to Orion in richness. In particular, the Hyades likely contained a number of $\mathrm{O}$ stars capable of photoevaporating disks around young solar mass stars, as is seen to occur in Orion. The estimated lifetimes of disks in Orion are only 100,000 years, probably too short to form Jupiter mass planets. Perhaps stars on the outskirts of the cluster, sufficiently far from the $\mathrm{O}$ stars, might escape this stripping allowing them to form planets.

A program to obtain spectroscopic metallicities for a volume limited sample of hundreds of solar type stars would be time consuming, but the information it could provide for the frequency of terrestrial type planets would be invaluable. The alternative seems to be space based observations from telescopes, which will be orders of magnitude more expensive.

\section{Acknowledgements}

I would like to thank the conference organizers for the invitation to speak, and in particular to Andrea Dupree for her great patience and gentle proddings. This work was supported by NSERC of Canada, and by the Canada Research Chairs program. 


\section{References}

Boesgaard, A. M. \& Tripicco, M. J. 1986, ApJ, 302, L49

Ferlet, R., Vidal-Madjar, A., \& Hobbs, L. M. 1987, A\&A, 185, 267

Gratton, R. G., Bonanno, G., Claudi, R. U., Cosentino, R., Desidera, S., Lucatello, S., \& Scuderi, S. 2001, A\&A, 377, 123

Henney, C. J. \& Ulrich, R. K. 1998, Structure and Dynamics of the Interior of the Sun and Sun-like Stars SOHO 6/GONG 98 Workshop Abstract, June 1-4, 1998, Boston, Massachusetts, p. 473, 6, 473

Israelian, G., Santos, N. C., Mayor, M., \& Rebolo, R. 2001, Nature, 411, 163

Israelian, G., Santos, N. C., Mayor, M., \& Rebolo, R. 2003, A\&A, 405, 753

Laws, C. \& Gonzalez, G. 2001, ApJ, 553, 405

Murray, N., Chaboyer, B., Arras, P., Hansen, B., \& Noyes, R. W. 2001, ApJ, 555,801

Paulson, D. B., Sneden, C., \& Cochran, W. D. 2003, AJ, 125, 3185

Petit, J., Morbidelli, A., \& Chambers, J. 2001, Icarus, 153, 338

Reddy, B. E., Lambert, D. L., Laws, C., Gonzalez, G., \& Covey, K. 2002, MNRAS, 335, 1005

Smith, V. V., Cunha, K., \& Lazzaro, D. 2001, AJ, 121, 3207

Spangler, C., Sargent, A. I., Silverstone, M. D., Becklin, E. E., \& Zuckerman, B. 2001, ApJ, 555, 932

Takeda, Y. et al. 2001, PASJ, 53, 1211

Winnick, R. A., Demarque, P., Basu, S., \& Guenther, D. B. 2002, ApJ, 576, 1075

Wolszczan, A. \& Frail, D. A. 1992, Nature, 355, 145 